\title{
Resistance to Cetuximab in EGFR-Overexpressing Esophageal Squamous Cell Carcinoma Xenografts Due to FGFR2 Amplification and Overexpression
}

\author{
Yi Zhang ${ }^{1}$, Tiecheng Pan $^{2}$, Xiaoxuan Zhong ${ }^{2}$, and Cai Cheng ${ }^{2, *}$ \\ ${ }^{1}$ Department of Surgery, The Third Affiliated Hospital of Jianghan University, \\ 259 Baixiu Street, Huangpi District, Wuhan 430300, China \\ ${ }^{2}$ Division of Cardiothoracic Surgery, Tongji Hospital of Tongji Medical College, Huazhong University of Science and \\ Technology, Pan Tiecheng: 1095 Jiefang Ave, Wuhan 430030, China
}

Received June 16, 2014; Accepted July 24, 2014

\begin{abstract}
Esophageal carcinoma is one of the most virulent malignant diseases and a major cause of cancer-related deaths worldwide. Despite improvements in surgical techniques and perioperative management and surgery combined with chemotherapy and/or radiotherapy, the prognosis of esophageal squamous cell carcinoma (ESCC) at an advanced stage remains poor. ESCC shows a relatively high incidence of EGFR (50\%-70\%), and the humanized monoclonal antibody $(\mathrm{mAb})$ cetuximab against EGFR has been undergoing clinical development. However, all responding patients eventually developed acquired resistance to cetuximab. In the current study, we described a cetuximab-sensitive ESCC xeongraft model that developed resistance to cetuximab as a result of FGFR2 gene amplification and overexpression. Inhibition of FGFR2 signaling in this xenograft model restored its sensitivity to cetuximab. The antitumor effect may be induced by inhibition of AKT phosphorylation. These findings suggest that combination therapy including cetuximab and FGFR2 inhibition may be a promising strategy to treat ESCC.
\end{abstract}

Keywords: EGFR, FGFR2, cetuximab, esophageal squamous cell carcinoma

\section{Introduction}

Despite extensive investigations of therapeutic improvements in surgical techniques, carcinoma of the esophagus remains a devastating disease and one of the most frequent causes of cancer-related deaths in the world $(1,2)$. The vast majority of esophageal cancers occur as either squamous cell carcinoma in the middle or upper third of the esophagus (ESCC) or as adenocarcinoma in the distal third or esophagogastric junction, with other tumor types occurring only very rarely. Most patients with esophageal cancer in Asian countries have squamous cell carcinoma, while most of those in Western countries have adenocarcinoma (3). However, the prognosis of ESCC at an advanced stage remains poor, half of all patients develop recurrences within a few

*Corresponding author. cai_cheng111@126.com

Published online in J-STAGE

doi: 10.1254/jphs.14150FP years after surgery and the 5-year survival rate is only approximately $50 \%(4-6)$.

The ERBB proteins are cell membrane tyrosine kinase receptors. This family is composed of ERBB1/EGFR, ERBB2/HER2, ERBB3/HER3, and ERBB4/HER4, which play essential roles in promoting cell growth, migration, differentiation, proliferation, and survival. Each receptor has an extracellular domain, lipophilic transmembrane domain, and an intracellular tyrosine kinase domain. Two major signaling routes activated by ERBB-receptor family members are the RAS-RAF-ERK and phosphatidylinositol 3-kinase (PI3K)-AKT pathways $(7,8)$. Approximately $50 \%-70 \%$ of ESCC tumors express EGFR protein when examined using immunohistochemistry (IHC), while $15 \%-28 \%$ of specimens exhibit $E G F R$ gene amplification when examined using fluorescence in situ hybridization (FISH) $(9,10)$. Therefore, for ESCC patients, novel therapies such as moleculartargeted therapy, including small molecule tyrosine kinases inhibitors (TKIs) and humanized monoclonal 
antibodies (mAbs) are very much needed.

Cetuximab (Erbitux ${ }^{\mathrm{TM}}$ ) is an anti-EGFR monoclonal antibody used for the treatment of metastatic colorectal cancer, metastatic non-small cell lung cancer, and head and neck cancer and is in clinical trials for esophageal cancers $(11,12)$. Cetuximab is thought to act via blockage of ligand binding and receptor dimerization, receptor down-regulation, and apoptosis induction (13). Despite the success of using cetuximab to treat patients harboring specific $E G F R$ mutations, all responding patients eventually developed acquired resistance to cetuximab $(14,15)$. However, the mechanism of acquired resistance to cetuximab in ESCC is unclear. In the current study, we described a cetuximab-sensitive ESCC xeongraft model that developed resistance to cetuximab and tried to investigate the underlying mechanism.

\section{Materials and Methods}

\section{Cell lines}

Human esophageal squamous cell carcinoma cell line TE-6 was obtained from the Health Science Research Resources Bank (Osaka), which harbors EGFR high expression (16). Cells were cultured in Dulbecco's modified Eagle's medium (DMEM; Gibco, Grand Island, NY, USA) supplemented with $10 \%$ fetal bovine serum (FBS, Gibco), penicillin and streptomycin (ICN Biomedicals, Inc., Costa Mesa, CA, USA), and sodium pyruvate (Bioproducts, Middletown, MD, USA) and maintained at $37^{\circ} \mathrm{C}$ with $5 \% \mathrm{CO}_{2}$ in a humidified atmosphere.

\section{Reagents}

The human-mouse chimeric anti-EGFR monoclonal antibody cetuximab was supplied by ImClone Systems, Inc. (New York, NY, USA). A selective pan-FGFR inhibitor NVP-BGJ398 was purchased from Sellech Chemicals (Houston, TX, USA). Phospho-EGFR (Tyr1068, p-EGFR), phospho-AKT (Ser473, p-AKT), phospho-ERK1/2 (Thr202/Tyr204, p-ERK1/2), phosphoFGFR2 (Tyr653/654, p-FGFR2), EGFR, AKT, FGFR2, and ERK1/2 antibodies were purchased from Santa Cruz Biotechnology, Inc. (Santa Cruz, CA, USA). The other chemicals used in this study were of analytical reagent grade. Tissue lysis buffer and phosphatase inhibitor cocktails were purchased from Sigma (St. Louis, MO, USA).

\section{Cell viability assay}

The CellTiter-Glo ${ }^{\circledR}$ luminescent cell viability assay (Promega, Madison, WI, USA) was used to determine the effect of cetuximab on cellular proliferation in TE-6 cell lines. Ten thousand cells were seeded in 96-well plates. After $24 \mathrm{~h}$ of attachment to the bottom of the plates, cells were treated by a serial dilution of cetuximab for $72 \mathrm{~h}$. Then, $50 \mu \mathrm{l}$ of the CellTiter-Glo reagent was added directly into each well and incubated for $10 \mathrm{~min}$. The plates were read by a GloMax ${ }^{\circledR}$ 96-microplate luminometer (Promega, Madison, WI, USA) after incubation to monitor the luminescence signal generated by the luciferase-catalyzed reaction of luciferin and ATP. The $\mathrm{IC}_{50}$ value was determined using GraphPad Prism software (version 5.0; La Jolla, CA, USA). This was defined as the treatment concentration at which $50 \%$ reduction in cellular proliferation was observed.

\section{TE-6 xenografts establishment and efficacy study in vivo}

Female athymic BALB/c nude mice, 6 weeks of age, were purchased from Vital River (Beijing, China). Mice were maintained under super pathogen-free conditions and housed in barrier facilities on a 12-h light/dark cycle, with food and water ad libitum. All animal experiments were performed in accordance with protocols approved by the Huazhong University Experimental Animal Care and Use Committee. Tumor volume was calculated using the following formula: tumor volume $=$ (length $\times$ width 2 ) $/ 2$, where length and width are the longest and shortest dimensions of the tumor, respectively. Mice were injected subcutaneously (s.c.) with $5 \times 10^{6}$ TE-6 cells, which had been resuspended in $200 \mu \mathrm{l}$ of matrigel (BD Biosciences, Milan, IT). When tumor volume reached $180-200 \mathrm{~mm}^{3}$, the mice were randomly divided in 4 groups (10 mice per group). The tumor volume and body weight in each group were well balanced. Four groups were twice weekly given an intraperitoneal (i.p.) injection of PBS or 10, 20, or $30 \mathrm{mg} / \mathrm{kg}$ cetuximab, respectively, for 3 weeks.

\section{Cetuximab-resistant TE-6 xenograft establishment and efficacy study in vivo}

Mice were injected subcutaneously (s.c.) with $5 \times 10^{6}$ TE- 6 cells, which had been resuspended in $200 \mu$ of matrigel. When tumor volume reached $180-200 \mathrm{~mm}^{3}$, 10 mice in each group were treated with PBS or $30 \mathrm{mg} / \mathrm{kg}$ cetuximab twice weekly by i.p. injection. Tumors were monitored for the development of cetuximab resistance, defined as marked tumor growth in the presence of continued cetuximab therapy. Approximately 20 weeks following the injection, the tumors $(2 \mathrm{~cm}$ in diameter) were aseptically resected from the cetuximab-resistant group and minced into $3 \times 3 \times 3 \mathrm{~mm}$ pieces. Host mice were then anesthetized with isoflurane, and a piece of tumor was implanted into the left flank of each mouse. When tumor volume reached $180-200 \mathrm{~mm}^{3}$, mice were randomly divided in 4 groups (10 mice per group). The tumor volume and body weight in each group were 
balanced. The dose regiment was designed as follows: group 1 was twice weekly given an i.p. injection with PBS and once-daily oral dosed with acetic acid buffer pH 4.6 / PEG300. Group 2 was twice weekly given an i.p. injection with $30 \mathrm{mg} / \mathrm{kg}$ cetuximab. Group 3 was once-daily oral dosed (p.o.) with $15 \mathrm{mg} / \mathrm{kg}$ NVP-BGJ398, and group 4 was treated with the combination of cetuximab with BGJ398 for 3 weeks. NVP-BGJ398 was dissolved in acetic acid buffer pH 4.6 / PEG300 (17).

\section{Detection of FGFR2 copy number and expression by microarray}

The GeneChip Human Genome SNP 6.0 and U133 plus 2.0 arrays (Affymetrix, Santa Clara, CA, USA) were used to analyze the genomic gene copy number and gene expression between cetuximab-sensitive and resistant TE-6 tumors. Gene profiling comparison was performed by calculating the fold change of the copy number and gene expression between these tumors. The genes changed in both copy number and expressions were determined for further bioinformatics analysis.

\section{Western blot analysis}

The expressions of p-EGFR, p-ERK1/2, p-AKT, and p-FGFR2 in tumor tissues were examined by western Blotting. The tumor tissues were resected at $2 \mathrm{~h}$ after the last treatment with cetuximab and/or NVP-BGJ398 on day 21 of the efficacy studies. The total protein was isolated from tumor samples with tissue lysis buffer (Bio-Rad Laboratories, Hercules, CA, USA) supplemented with phosphatase inhibitor cocktail. Protein of the tumor tissues was extracted by RIPA buffer supplemented with phosphatase inhibitor cocktails. The protein concentration of the supernatant was determined by Bio-Rad protein assay reagent (Bio-Rad Laboratories). Equal amounts of protein were separated by sodium dodecyl sulfate/polyacrylamidegel electrophoresis (SDS/ PAGE) on $10 \%$ gels; blotted on a nitrocellulose membrane; and probed with p-EGFR, p-ERK1/2, p-AKT, $\mathrm{p}$-FGFR2, and $\beta$-actin rabbit monoclonal antibody. The blots were then incubated with goat anti-rabbit (HRP) and detected by chemiluminescence.

\section{Statistical analyses of the data}

All results and data were confirmed in at least 3 separate experiments. Data are expressed as means \pm S.D. and were analyzed by Student's $t$-test using Statistics Package for Social Science (SPSS) software (version 13.0; SPSS, Chicago, IL, USA). Differences were considered to be statistically significant when the $P$-value was $<0.05$.

\section{Results}

Effect of cetuximab on viability of TE-6 cells in vitro

Before evaluating the effect of cetuximab treatment on TE-6 xenograft models in nude mice, the sensitivity of the cell line to cetuximab was evaluated in vitro. Cell proliferation was analyzed by the CellTiter-Glo ${ }^{\circledR}$ luminescent cell viability assay in cells treated with $0,0.01,0.1,1,10$, and $100 \mu \mathrm{g} / \mathrm{ml}$ of cetuximab for $72 \mathrm{~h}$. Cetuximab concentration-dependently induced TE- 6 cell growth. By $72 \mathrm{~h}$ after treatment with $10 \mu \mathrm{g} / \mathrm{ml}$ cetuximab, the cell viability was only $22 \%$, and the $\mathrm{IC}_{50}$ for cetuximab treatment was $2.4 \mu \mathrm{g} / \mathrm{ml}$ (Fig. 1).

Tumor growth inhibition effect of cetuximab on TE-6 xenografts and cetuximab-resistant TE-6 xenografts establishment

To investigate the tumor growth inhibition effect of cetuximab on TE- 6 xenografts in vivo, TE-6 tumor bearing mice were injected i.p. twice a week with 10 , 20 , or $30 \mathrm{mg} / \mathrm{kg}$ cetuximab for up to 3 weeks. As shown in Fig. 2, A and B, treatment with $20 \mathrm{mg} / \mathrm{kg}$ cetuximab was able to inhibit tumor growth of TE-6 xenografts, but $10 \mathrm{mg} / \mathrm{kg}$ cetuximab could slightly inhibit tumor growth, whereas $30 \mathrm{mg} / \mathrm{kg}$ cetuximab treatment could almost prevent tumor growth entirely. In the other cetuximab-resistant TE-6 xenograft establishment study, approximately 20 weeks following the $30 \mathrm{mg} / \mathrm{kg}$ cetuximab i.p. injection, some TE-6 xenografts acquired resistance to cetuximab (Fig. 2C). The cetuximab treatments were well tolerated by mice, with no weight loss or other signs of acute or delayed toxicity (Fig. 2D).

FGFR2 was highly amplified and expressed in the cetuximab-resistant TE-6 xenografts

As shown in Fig. 2C the tumor growth could not be inhibited by $30 \mathrm{mg} / \mathrm{kg}$ cetuximab after 20 weeks'

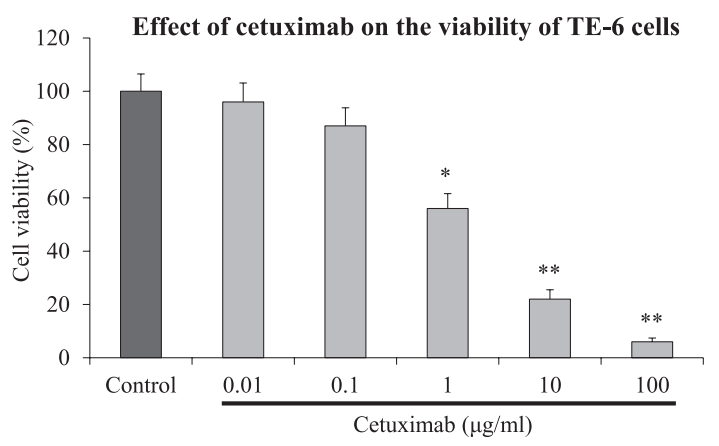

Fig. 1. Anti-proliferative effect of cetuximab in TE-6 cell line. Cells were treated with varying concentrations of cetuximab for $72 \mathrm{~h}$. Doses ranged from 0.01 to $100 \mu \mathrm{g} / \mathrm{ml}$. Mean \pm S.D., $\mathrm{n}=3$. $* P<0.05$, ** $P<0.01$ vs. control group. 
A

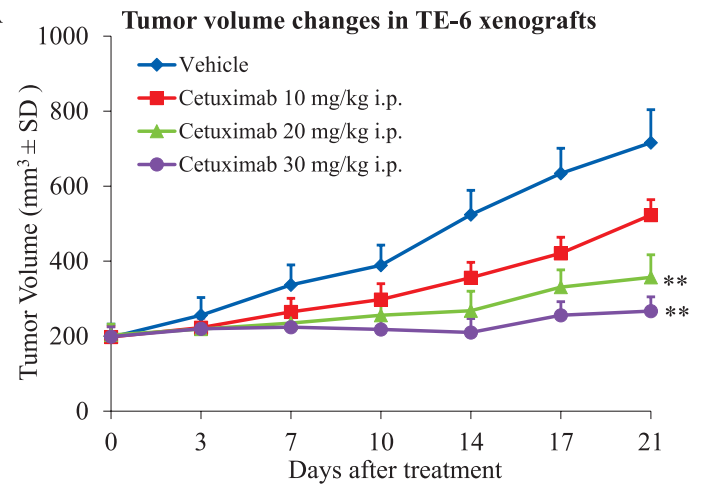

B

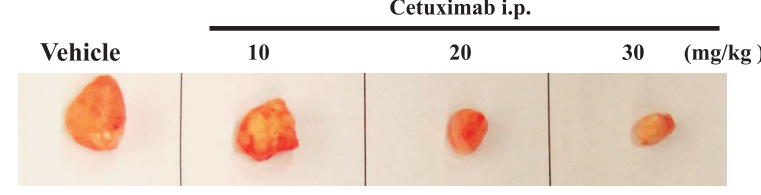

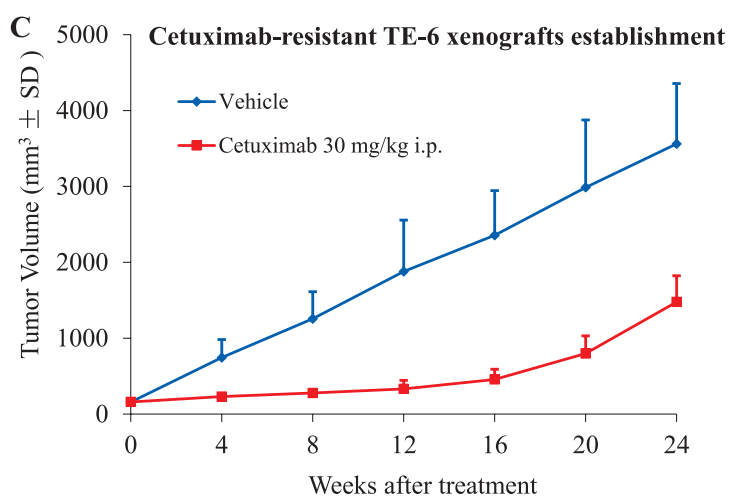

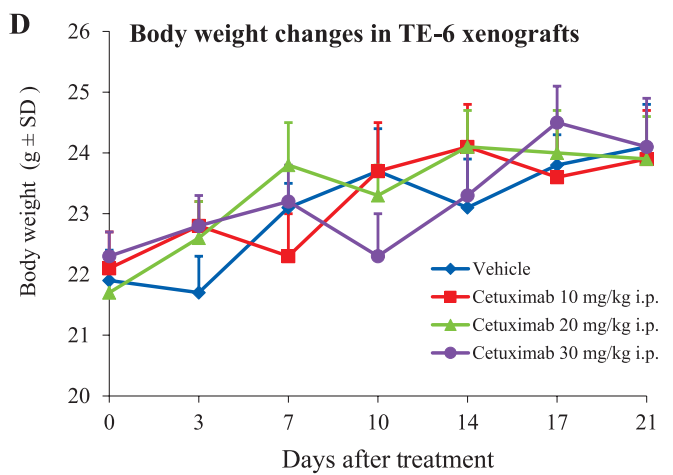

Fig. 2. Anti-tumor activity of cetuximab in TE-6 xenografts and cetuximab-resistant TE-6 xenograft establishment. A) Nude mice-bearing TE-6 tumors were i.p. injected twice weekly with 10, 20, or $30 \mathrm{mg} / \mathrm{kg}$ cetuximab for 3 weeks. B) Tumors were resected from nude mice on day 21. C) Mice were treated with $30 \mathrm{mg} / \mathrm{kg}$ cetuximab, twice weekly by i.p. injection, for 24 weeks. D) Body weight was measured on the indicated days. Mean \pm S.D., $\mathrm{n}=10 . * * P<0.01$ vs vehicle group.

treatment. To explore the mechanism of the resistance to cetuximab, we compared the genome-wide gene profile between cetuximab-sensitive and cetuximabresistant tumors and found that FGFR2 was highly amplified and expressed in the cetuximab-resistant TE-6 xenografts, whereas in the cetuximab-sensitive TE-6 xenografts, the $F G F R 2$ was expressed at a normal level and not amplified (Fig. 3: A, B). These results were confirmed by the western blot analysis (Fig. 5A). In addition, the gene copy number and expression of $E G F R$, HER 2, HER 3, MET, and KRAS were also analyzed, and there was no significant difference between cetuximabsensitive and resistant models. According to the results we proposed that FGFR2 may be related to the acquired resistance to cetuximab, and further studies were needed to confirm this.

Combination cetuximab with NVP-BGJ398 has a synergistic tumor growth inhibition effect on cetuximabresistant TE-6 xenografts

Since FGFR2 was highly amplified and expressed in the cetuximab-resistant TE- 6 xenografts, we combined cetuximab with the selective pan-FGFR kinase inhibitor NVP-BGJ398 to treat cetuximab-resistant TE-6 xeno-
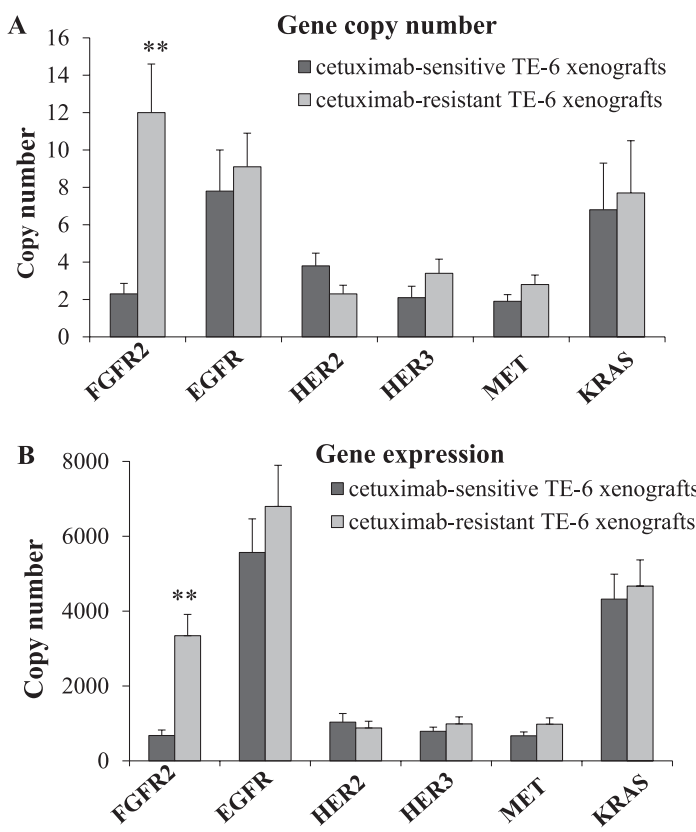

Fig. 3. FGFR2 was highly amplified and expressed in cetuximabresistant xenografts. Cetuximab-sensitive and resistant tumors were resected from xenografts, respectively, and then analyzed by the GeneChip Human Genome SNP 6.0 (A) and U133 plus 2.0 arrays (B). Mean \pm S.D., $\mathrm{n}=5$. ${ }^{* *} P<0.01$ vs. cetuximab-sensitive xenografts. 

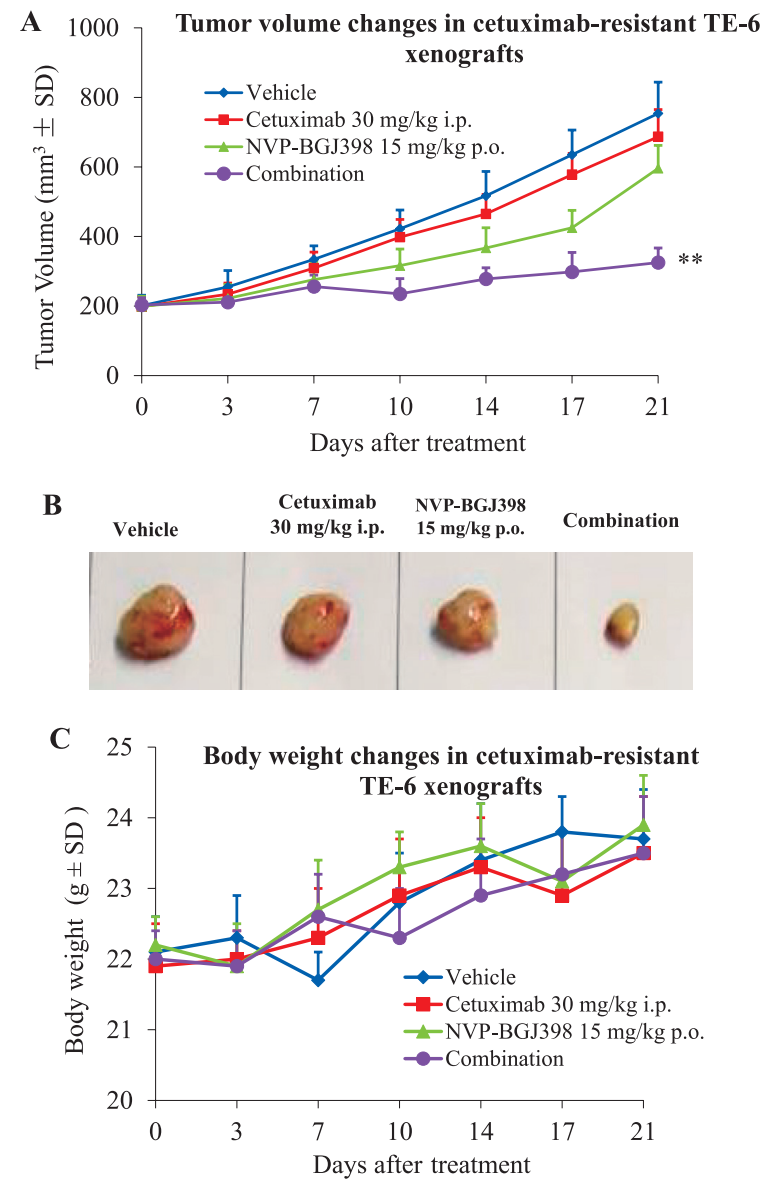

Fig. 4. Anti-tumor activity of cetuximab in combination with NVPBGJ398 in cetuximab-resistant TE-6 xenografts. A) Nude mice bearing resistant TE-6 tumors were treated with $30 \mathrm{mg} / \mathrm{kg}$ cetuximab twice weekly by i.p. injection, $15 \mathrm{mg} / \mathrm{kg}$ NVP-BGJ398 once daily by oral administration, or a combination therapy for 3 weeks. B) Tumors were resected from nude mice on day 21. C) Body weight was measured on the indicated days. Mean \pm S.D., $\mathrm{n}=10 . * * P<0.01$ vs. vehicle group.

grafts (15). As shown in Fig. 4, A and B, treatment with $30 \mathrm{mg} / \mathrm{kg}$ cetuximab was not able to inhibit tumor growth in cetuximab-resistant TE- 6 xenografts, and $15 \mathrm{mg} / \mathrm{kg}$ NVP-BGJ398 could slightly inhibit tumor growth. In contrast, the combined treatments with the two drugs almost completely inhibited tumor growth at the end of the 3 weeks of therapy. Single agent and combination treatment protocols were well tolerated by mice, with no weight loss or other signs of acute or delayed toxicity (Fig. 4C).

Effects of cetuximab and/or NVP-BGJ398 on signaling transduction pathways in cetuximab-sensitive or resistant TE-6 tumor models

To assess the impact of both inhibitors on downstream molecules of the PI3K and ERK pathways, we used western blot analysis to observe phosphorylation status and total protein expression in tumor tissues. The results showed that both cetuximab-sensitive and resistant TE-6 xenografts highly expressed p-EGFR and EGFR, whereas the expressions of p-FGFR2 and FGFR2 were only observed in cetuximab-resistant TE-6 xenografts (Fig. 5A). In addition, p-EGFR and p-ERK1/2 appeared to be significantly inhibited by cetuximab in both cetuximab-sensitive and resistant TE-6 xenografts, whereas the protein level of p-AKT was only inhibited in cetuximab-sensitive TE-6 xenografts but not in cetuximab-resistant xenografts (Fig. 5: B and C). The expression of p-FGFR 2 would be significantly inhibited by NVP-BGJ398 in cetuximab-resistant xenografts. Combination treatment with cetuximab and NVPBGJ398 showed greater inhibition p-AKT than observed in the vehicle group or mice treated with each inhibitor alone in vivo (Fig. 5C).

\section{Discussion}

Although advances have been made in cancer treatment with the development of selective molecular targeted therapies, several relevant issues for their optimal and effective use remain unsolved. In the current study, we described a cetuximab-sensitive ESCC tumor model that developed resistance to cetuximab as a result of FGFR2 gene amplification and overexpression by comparing the genome-wide gene profile of the sensitive and resistant xenografts. Unexpectedly, the protein level of FGFR2 was found to be highly expressed in cetuximab-resistant TE-6 xenografts by western blot analysis. Further study showed that inhibition of the FGFR2 pathway by NVP-BGJ398 could recover the sensitivity to cetuximab in the resistant xenografts, which indicated that FGFR2 contributed to cetuximab resistance in the ESCC tumor model. These findings support a possible role for cetuximab and NVP-BGJ398 combination therapy in the treatment of ESCC and may help further the understanding of the complex interactions and downstream signaling of EGFR and FGFR2 receptors in ESCC.

A number of articles have reported on the mechanisms of acquired resistance to cetuximab. Wheeler et al. reported that lung cancer cells developing acquired resistance to cetuximab exhibited increased steady-state EGFR expression secondary to alterations in trafficking and degradation. In addition, cetuximab-resistant cells manifested strong activation of HER2, HER3, and MET (18). Further, Misale et al. reported that molecular alterations (in most instances point mutations) of KRAS are causally associated with the onset of acquired resistance to cetuximab treatment in colorectal cancers (19). 


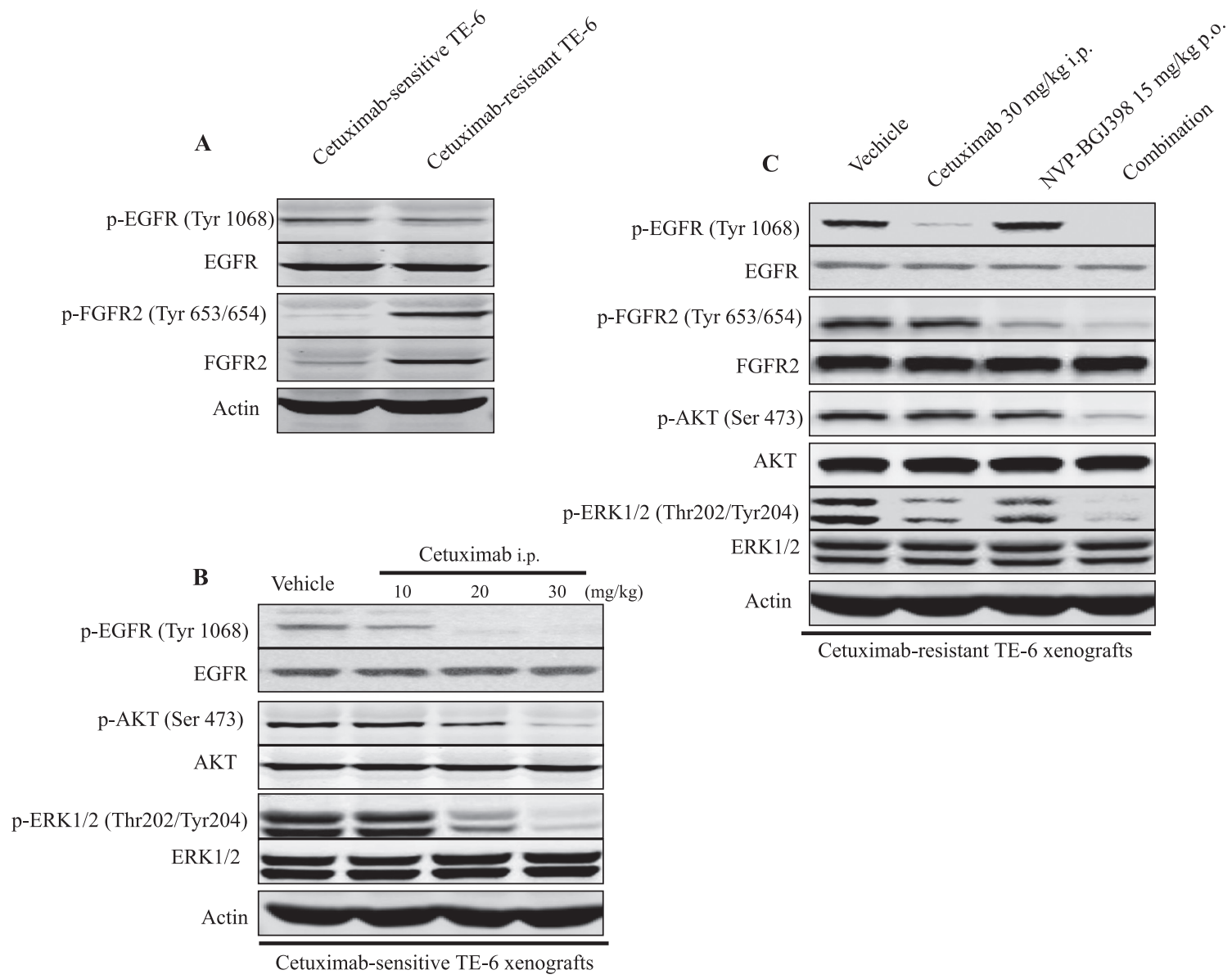

Fig. 5. Effects of cetuximab or/and NVP-BGJ398 on signaling transduction pathways in cetuximab-sensitive or resistant TE-6 tumor models. A) The expression levels of p-EGFR, EGFR, p-FGFR2, and FGFR2 were analyzed by western blotting in cetuximab-sensitive or resistant TE-6 tumors. B and C) The tumor tissues were resected at $2 \mathrm{~h}$ after the last treatment with cetuximab or/and NVP-BGJ398 on day 21 of each efficacy study and then subjected to western blotting for detection of p-EGFR, p-FGFR2, p-AKT, and p-ERK expressions.

While in our study, there was no significant difference regarding EGFR, HER2, HER3, MET, and KRAS gene copy number and expression between cetuximabsensitive and resistant models, which indicated FGFR2 amplification and overexpression may contribute to cetuximab resistance in ESCC tumor model. More interestingly, the protein level of p-AKT was inhibited only in cetuximab-sensitive TE-6 xenografts but not in cetuximab-resistant xenografts. Unexpected, combination treatment could completely inhibit p-AKT expression in both cetuximab-sensitive and resistant xenografts. These results indicated that the contribution of FGFR2 to cetuximab resistance might depend on AKT signaling transduction, and further investigations are needed to confirm this.

RAS/RAF/ERK signaling plays a critical role in a wide range of biological processes such as cell proliferation and differentiation, cell migration and survival, and cellular metabolism $(20-23)$. In the current study, the phosphorylation level of ERK1/2 in both cetuximabsensitive and -resistant xenografts would be significantly inhibited by cetuximab. Thus, we deduce that the mechanisms of acquired resistance to cetuximab might not depend on RAS/RAF/ERK signaling transduction. Although the mechanisms of acquired resistance to cetuximab have been studied in other cancer types, to our knowledge the present study is the first to explore this mechanism in ESCC.

In conclusion, cetuximab-sensitive TE-6 xenograft model developed resistance to cetuximab as a result of FGFR2 gene amplification and overexpression. Combination of cetuximab and NVP-BGJ398 exerted significant synergistic antitumor effect on the cetuximabresistant xenografts in vivo. Then, the antitumor effect may be induced by the inhibition of AKT phosphorylation. These findings suggest that combination therapy 
including cetuximab and NVP-BGJ398 may be a promising strategy to treat acquired cetuximab-resistant ESCC.

\section{Acknowledgments}

The study was funded by the grant "Experimental Study of the Splenic Artery Targeted Removal of Activated T Cells to Inhibit Acute Rejection", 2012FFB03401, longitudinal support project by the Science and Technology Department of Hubei Province.

The authors thank Junjin Xu and Lin Li for bioinformatics analysis of the GeneChip Human Genome SNP 6.0 and U133 plus 2.0 arrays' data.

\section{Conflicts of Interest}

The authors declare no competing interests.

\section{References}

1 Dawsey SP, Tonui S, Parker RK, Fitzwater JW, Dawsey SM, White RE, et al. Esophageal cancer in young people: a case series of 109 cases and review of the literature. PLoS One. 2010; 5:e14080.

2 Ferlay J, Shin H, Bray F, Forman D, Mathers C, Parkin D. Estimates of worldwide burden of cancer in 2008: GLOBOCAN 2008. Int J Cancer. 2010;127:2893-2917.

3 Siegel R, Ward E, Brawley O, Jemal A. Cancer statistics, 2011: the impact of eliminating socioeconomic and racial disparities on premature cancer deaths. CA Cancer J Clin. 2011;61:212-236.

4 Pohl H, Sirovich B, Welch HG. Esophageal adenocarcinoma incidence: are we reaching the peak? Cancer Epidemiol Biomarkers Prev. 2010;19:1468-1470.

5 Nakagawa S, Kanda T, Kosugi S, Ohashi M, Suzuki T, Hatakeyama $\mathrm{K}$. Recurrence pattern of squamous cell carcinoma of the thoracic esophagus after extended radical esophagectomy with three-field lymphadenectomy. J Am Coll Surg. 2004;198:205-211.

6 Stiles BM, Nasar A, Mirza FA, Lee PC, Paul S, Port JL, et al. Worldwide Oesophageal Cancer Collaboration guidelines for lymphadenectomy predict survival following neoadjuvant therapy. Eur J Cardiothorac Surg. 2012;42:659-664.

7 Olayioye MA, Neve RM, Lane HA, Hynes NE. The ErbB signaling network: receptor heterodimerization in development and cancer. EMBO J. 2000;19:3159-3167.

8 Normanno N, Bianco C, De Luca A, Maiello MR, Salomon DS. Target-based agents against ErbB receptors and their ligands: a novel approach to cancer treatment. Endocr Relat Cancer. 2003;10:1-21.

9 Hanawa M, Suzuki S, Dobashi Y, Yamane T, Kono K, Enomoto $\mathrm{N}$, et al. EGFR protein overexpression and gene amplification in squamous cell carcinomas of the esophagus. Int $\mathrm{J}$ Cancer. 2006;118:1173-1180.
10 Sunpaweravong P, Sunpaweravong S, Puttawibul P, Mitarnun W, Zeng C, Barón AE, et al. Epidermal growth factor receptor and cyclin D1 are independently amplified and overexpressed in esophageal squamous cell carcinoma. J Cancer Res Clin Oncol. 2005;131:111-119.

11 Friedlander E, Barok M, Szollosi J, Vereb G. ErbB-directed immunotherapy, antibodies in current practice and promising new agents. Immunol Lett. 2008;116:126-140.

12 Schmitz KR, Ferguson KM. Interaction of antibodies with ErbB receptor extracellular regions. Exp Cell Res. 2009;315:659-670.

13 Kullmann F, Hollerbach S, Dollinger MM, Harder J, Fuchs M, Messmann $\mathrm{H}$, et al. Cetuximab plus gemcitabine/oxaliplatin (GEMOXCET) in first-line metastatic pancreatic cancer: a multicentre phase II study. Br J Cancer. 2009;100:1032-1036.

14 Rampias T, Giagini A, Siolos S, Matsuzaki H, Sasaki C, Scorilas A, et al. RAS/PI3K Crosstalk and Cetuximab Resistance in Head and Neck Squamous Cell Carcinoma. Clin Cancer Res. 2014;20: 2933-2946.

15 Pietrantonio F, Maggi C, Di Bartolomeo M, Facciorusso MG, Perrone F, Testi A, et al. Gain of ALK gene copy number may predict lack of benefit from anti-EGFR treatment in patients with advanced colorectal cancer and RAS-RAF-PI3KCA wildtype status. PLoS One. 2014;9:e92147.

16 Yamazaki M, Yamashita Y, Kubo N, Yashiro M, Ohira M, Ako E, et al. Concurrent biological targeting therapy of squamous cell carcinoma of the esophagus with cetuximab and trastuzumab. Oncol Rep. 2012;28:49-54.

17 Guagnano V, Kauffmann A, Wohrle S, Stamm C, Ito M, Barys L, et al. FGFR genetic alterations predict for sensitivity to NVPBGJ398, a selective pan-FGFR inhibitor. Cancer Discov. 2012;12:1118-1133.

18 Wheeler DL, Huang S, Kruser TJ, Nechrebecki MM, Armstrong EA, Benavente S, et al. Mechanisms of acquired resistance to cetuximab: role of HER (ErbB) family members. Oncogene. 2008;27:3944-3956.

19 Misale S, Yaeger R, Hobor S, Scala E, Janakiraman M, Liska D, et al. Emergence of KRAS mutations and acquired resistance to anti-EGFR therapy in colorectal cancer. Nature. 2012;486: 532-536.

20 McKay MM, Morrison DK. Integrating signals from RTKs to ERK/MAPK. Oncogene. 2007;9:3113-3121.

21 Tidyman WE, Rauen KA. The RASopathies: developmental syndromes of Ras/MAPK pathway dysregulation. Curr Opin Genet Dev. 2009;9:230-236.

22 Karnoub AE, Weinberg RA. Ras oncogenes: split personalities. Nat Rev Mol Cell Biol. 2008;9:517-531.

23 Liu Y, Shi QF, Ye YC, Tashiro S, Onodera S, Ikejima T. Activated $\mathrm{O} 2(\bullet-)$ and $\mathrm{H} 2 \mathrm{O} 2$ mediated cell survival in SU11274-treated non-small-cell lung cancer A549 cells via c-Met-PI3K-Akt and c-Met-Grb2/SOS-Ras-p38 pathways. J Pharmacol Sci. 2012;119: 150-159. 
